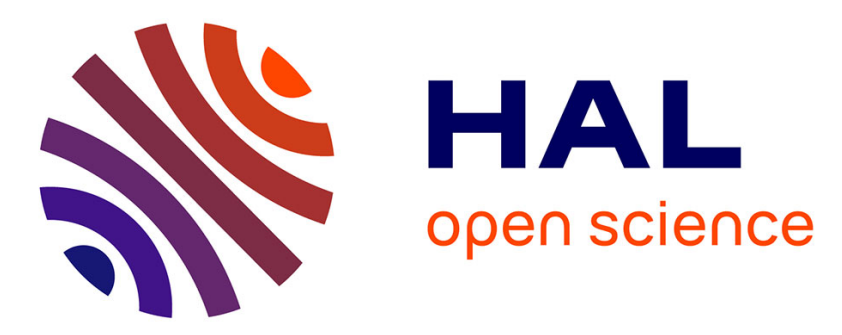

\title{
L'insight et les croyances relatives aux médicaments influencent l'observance médicamenteuse dans la schizophrénie
}

\author{
C.M. Paillot, P. Ingrand, I. Ingrand, N. Jaafari
}

\section{- To cite this version:}

C.M. Paillot, P. Ingrand, I. Ingrand, N. Jaafari. L'insight et les croyances relatives aux médicaments influencent l'observance médicamenteuse dans la schizophrénie. Annales Médico-Psychologiques, Revue Psychiatrique, 2011, 10.1016/j.amp.2011.06.011 . hal-00789300

\section{HAL Id: hal-00789300 https://hal.science/hal-00789300}

Submitted on 18 Feb 2013

HAL is a multi-disciplinary open access archive for the deposit and dissemination of scientific research documents, whether they are published or not. The documents may come from teaching and research institutions in France or abroad, or from public or private research centers.
L'archive ouverte pluridisciplinaire HAL, est destinée au dépôt et à la diffusion de documents scientifiques de niveau recherche, publiés ou non, émanant des établissements d'enseignement et de recherche français ou étrangers, des laboratoires publics ou privés. 


\section{Accepted Manuscript}

Title: L'insight et les croyances relatives aux médicaments influencent l'observance médicamenteuse dans la schizophrénie

Authors: C.M. Paillot, P. Ingrand, I. Ingrand, N. Jaafari

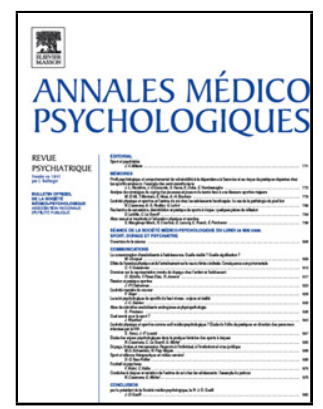

PII:

S0003-4487(11)00170-3

DOI: doi:10.1016/j.amp.2011.06.011

Reference: AMEPSY 1352

To appear in: Annales Médico-Psychologiques

Please cite this article as: Paillot CM, Ingrand P, Ingrand I, Jaafari N, L'insight et les croyances relatives aux médicaments influencent l'observance médicamenteuse dans la schizophrénie, Annales medio-psychologiques (2010), doi:10.1016/j.amp.2011.06.011

This is a PDF file of an unedited manuscript that has been accepted for publication. As a service to our customers we are providing this early version of the manuscript. The manuscript will undergo copyediting, typesetting, and review of the resulting proof before it is published in its final form. Please note that during the production process errors may be discovered which could affect the content, and all legal disclaimers that apply to the journal pertain. 


\title{
Communication
}

\section{L'insight et les croyances relatives aux médicaments influencent l'observance médicamenteuse dans la schizophrénie Predicting compliance in schizophrenia:}

The value of insight and attitudes toward treatment

\author{
C.M. Paillot ${ }^{a, b}$, P. Ingrand ${ }^{c}$, I. Ingrand ${ }^{c}$, N. Jaafari ${ }^{\text {d }}$ \\ ${ }^{a}$ Université Paris $X$, France \\ ${ }^{b}$ St. John's University, NY, USA
}

${ }^{c}$ Épidemiology et Biostatistics, INSERM CIC 802, CHU et Université de Poitiers, France

${ }^{d}$ Unité de recherche clinique intersectorielle en psychiatrie du Centre Hospitalier Henri Laborit, Inserm U802, CHU et Université de Poitiers, France

Auteur Correspondant : Nematollah Jaafari, Pavillon Toulouse, BP 587, Centre Hospitalier Henri Laborit, 86021 Poitiers, France

Adresse email : nemat.jaafari@ch-poitiers.fr

\section{Résumé}

L’identification des facteurs prédictifs de l'observance thérapeutique demeure un problème central dans la prise en charge de la schizophrénie. L’objectif de cette étude était d'évaluer si l'insight associé aux croyances relatives aux traitements permet une meilleure prédiction de l'observance.

Méthode - L’observance médicamenteuse, l’insight et les croyances relatives aux traitements de 98 patients présentant un diagnostic de schizophrénie ont été évalués la semaine suivant leur hospitalisation.

Résultats - Quarante-neuf pour cent des patients n’étaient pas observants. Les résultats de la régression logistique indiquent que l'observance médicamenteuse est davantage prédite par la sous-échelle de la DAI, le facteur 2 «J'ai besoin de prendre mes médicaments» lorsqu'il est combiné à la sous-échelle " conscience des troubles mentaux » de la SUMD $($ ROC AUC $=0,776)$.

Discussion - Il y aurait un recoupement conceptuel entre l'insight et le facteur 2 de la DAI : croyances relatives au besoin de recevoir un traitement antipsychotique. 
Conclusion - L'évaluation conjointe de l'insight clinique et des croyances relatives aux antipsychotiques optimise la prédiction de l'observance médicamenteuse dans la schizophrénie.

Mots clés: Croyances relatives aux médicaments; Observance médicamenteuse ; Schizophrénie

\section{Abstract}

Background - Even effective drugs are useless when not taken. The aim of this study is to assess whether attitudes toward treatment were a better predictor of compliance than insight in Schizophrenia.

Methods - 98 inpatients diagnosed with schizophrenia were evaluated within one week after being admitted to a psychiatric ward.

Results $-49 \%$ of patients were non compliant. Assessing jointly DAI and insight scores optimized the prediction of compliance to antipsychotic medication: results from a logistic regression indicated that compliance is better predicted by DAI factor 2 score “patient's assessment of need for medications” combined to SUMD G1 insight score "insight into mental disorder" (ROC AUC=0.776).

Discussion - It is possible that there is a conceptual overlap between patient's assessment of need for medication and clinical insight.

Conclusion - Clinical insight and attitudes toward treatment are stronger predictors of compliance when combined.

Keywords: Attitudes toward treatment; Compliance; Insight; Schizophrenia

\section{Introduction}

La plupart des patients souffrant de schizophrénie présentent une observance médicamenteuse partielle ou nulle [3,14]. Seul un tiers d'entre eux suivent leur traitement tel qu'il a été prescrit [10]. Le taux de non-compliance est de $25 \%$ après sept/dix jours, de $50 \%$ après un an, et va jusqu'à $75 \%$ après deux ans $[5,10]$. Ce défaut d'observance (partiel ou total) a des conséquences délétères sur l'évolution de la maladie et des conséquences économiques non négligeables [9,15]. La revue de la littérature de Velligan et al. rapporte que 
les croyances relatives aux médicaments jouent un rôle important dans la prédiction de l’observance [15]. Plus les croyances relatives aux médicaments sont négatives, moins le patient a de chances de prendre son traitement. Bien que l'insight et les croyances relatives aux médicaments soient des prédicteurs connus de l'observance médicamenteuse, la littérature relative à l'insight demeure contradictoire [8]. Étonnamment, peu d'études ont évalué la valeur prédictive de l'association entre l'insight et les croyances relatives aux traitements $[2,11,13]$. D’une part, ces études se révèlent parfois contradictoires, et, d'autre part, elles ont été réalisées uniquement en ambulatoire. Certaines confirment la valeur prédictive de l’insight, des croyances relatives aux médicaments [2,13] et de la sévérité de la maladie sur l'observance, mais ces données n’ont pas été répliquées [6,11]. À notre connaissance, le lien entre ces deux facteurs n’a jamais été étudié auprès de patients hospitalisés. Notre objectif est de déterminer si les croyances relatives aux traitements antipsychotiques sont un meilleur prédicteur de l'observance médicamenteuse que l'insight clinique, dans la Schizophrénie.

\section{Méthode}

Cette étude a été menée dans le service de psychiatrie adulte du Centre Hospitalier Henri Laborit à Poitiers, chez des patients présentant un diagnostic de schizophrénie (critères DSM-IV-TR). Les évaluations ont été réalisées lors de la première semaine suivant l’hospitalisation. L’insight Clinique a été évalué à l’aide de l'échelle d’hétéro-évaluation de la Conscience des Troubles (The Scale to assess Unawareness in Mental Disorder-SUMD [12]), seuls les items généraux ont été utilisés (item G1 : conscience de la maladie ; item G2 : conscience des effets thérapeutiques des médicaments; item G3 : conscience des conséquences sociales). Chaque item est coté de zéro à cinq, l’insight est d'autant plus faible que le score est bas. L’autoévaluation de l’insight a été évaluée par l'échelle d’Insight de Birchwood (Birchwood Insight Scale-IS [1]) composée de huit items, constituant trois souséchelles évaluant la conscience des symptômes, la conscience de la maladie et la conscience de la nécessité d'un traitement. Elle est cotée de zéro à douze, l'insight est d'autant plus faible que le score est élevé. Les croyances relatives aux antipsychotiques ont été évaluées à l'aide de l'Inventaire des croyances relatives aux médicaments The Drug Attitude Inventory (DAI10 [4]), qui est un questionnaire d'autoévaluation comprenant dix items. L'étendue du score total s'étend de - 10 à +10 . Un score négatif représente des croyances défavorables relatives aux médicaments et inversement. L’intensité de la symptomatologie psychotique a été 
évaluée à l'aide de la Positive and Negative Syndrome Scale (PANSS) [7]. Le relevé de l’observance per os était fondé sur un entretien clinique et la consultation du dossier médical ; celle du traitement injectable sur la réalisation de la dernière injection. L'analyse statistique a été réalisée à l’aide du logiciel SAS version 8 pour Windows ${ }^{\circledR}$.

\section{Résultats}

Quatre-vingt-dix-huit patients hospitalisés ont été inclus (les données sociodémographiques sont présentées dans le tableau 1). Chacun des participants était traité par antipsychotique (soit classique, soit atypique) au moment de l'évaluation, et $49 \%$ ne prenaient pas leur traitement au moment de l'étude. Le taux de patients observants traités par injectable (52\%) était comparable à celui des patients traités per os $(50 \%, p=0,83)$. L’intensité de la symptomatologie était significativement plus sévère pour les patients nonobservants (tableau 2). La corrélation du score global de la PANSS avec la DAI $(-0,35)$ indique que moins les symptômes des participants étaient sévères plus leurs croyances étaient favorables aux antipsychotiques. Il n’a pas été trouvé de corrélation entre le score aux symptômes négatifs de la PANSS et l'observance médicamenteuse.

Les résultats obtenus à la DAI-10 distinguent une double structure factorielle de l'échelle : le facteur 1 correspond aux « croyances relatives aux effets des antipsychotiques » (items un à cinq), et le facteur 2 aux «croyances relatives au besoin de recevoir un antipsychotique » (items six à dix ; Alpha de Cronbach =0,73). Le test de Mann-Whitney indique une différence statistiquement significative entre les patients observants et les patients non observants au score total à la DAI-10, notamment au facteur $2(\mathrm{p}<0,0001)$. La comparaison de l'insight met en évidence un insight significativement plus faible chez les non-observants (IS, DAI-10 total, facteur 2 de la DAI-10 - tableau 2). Les croyances des participants relatives à leur traitement étaient (DAI-10 total et le facteur 2 de la DAI-10) corrélées à leur niveau de conscience des effets thérapeutiques des antipsychotiques (item G2 de la SUMD) et à l'autoévaluation de la conscience des troubles mentaux (IS total). Plus l’insight évalué par les participants est bon, plus les croyances étaient favorables au traitement (coefficient de corrélation de Spearman entre le score total à l'IS et la DAI-10 [total, facteur 2] de 0,55 $\mathrm{p}=0,001)$. Ces résultats n’ont pas été confirmés par l’hétéro-évaluation de la conscience des troubles mentaux (Item G1 de la SUMD).

Les résultats de la régression logistique indiquent que l’observance médicamenteuse est mieux prédite par le facteur 2 de la DAI-10 « croyances relatives au besoin de recevoir un 
antipsychotique » associé au score d'Insight à l'item G1 de la SUMD «conscience des troubles mentaux » $($ ROC AUC = 0,776). Pris individuellement, le score total à la DAI-10 de même que le score au facteur 2 de la DAI-10 sont des prédicteurs moins puissants $($ ROC AUC $=0,663$ et ROC AUC $=0,726)$.

\section{Discussion}

Nos résultats confirment ceux rapportés par Freudenreich et al. en 2004 [2] et Rocca et al. en 2008 [13]. Deux éléments distinguent toutefois notre étude de celles précédemment citées : le fait que les patients étaient hospitalisés et la double évaluation de l’insight (auto et hétéro-évaluation). Nos résultats indiquent que les croyances relatives aux traitements sont fortement corrélées à la conscience du besoin de recevoir des antipsychotiques. En ce sens, ces deux dimensions sont utiles à évaluer, toutes deux permettant de prédire l'observance médicamenteuse dans la schizophrénie [3].

La structure factorielle de la DAI-10 suggère un possible recouvrement conceptuel entre l'évaluation par le patient du besoin d'antipsychotique (facteur 2) et l'insight clinique. S’agit-il en partie du même phénomène psychopathologique ? L’identification de ces deux facteurs de la DAI laisse à penser que les résultats contradictoires rapportés par les autres études sont probablement liés à des disparités méthodologiques et à une répartition hétérogène des patients présentant un score plus élevé au facteur 1 qu'au facteur 2 de la DAI.

La large taille de notre échantillon de patients ( $n=98)$, le fait que les patients étaient hospitalisés et la double évaluation de l'insight (auto et hétéro-évaluation), constituent les points forts de notre étude. L’absence de comparaison avec les propriétés psychométriques de la traduction française de la DAI-30, en revanche, est un point faible.

\section{Conclusion}

Nos résultats confirment les données présentes dans la littérature actuelle selon lesquelles l'insight clinique est un prédicteur de l'observance médicamenteuse et permettent d'affirmer qu'en associant l'évaluation des croyances relatives aux antipsychotiques à celle de la conscience de la maladie, on optimise cette prédiction.

\section{Conflit d'intérêt : à compléter par l'auteur}




\section{Références}

[1] Birchwood M, Smith J, Drury V, Healy J, Macmillan F, Slade M. A self-report insight scale for psychosis: reliability, validity and sensitivity to change. Acta Psychiatrica Scand 1994;89:62-7.

[2] Freudenreich O, Cather C, Evins AE, Henderson DC, Goff DC. Attitudes of schizophrenia outpatients toward psychiatric medications: relationship to clinical variables and insight. J Clin Psychiatry 2004;65:1372-6.

[3] Goff DC, Hill M, Freudenreich O. Strategies for improving treatment adherence in Schizophrenia and Schizoaffective disorder. J Clinical Psychiatry 2010;71:20-6.

[4] Hogan T, Awad AG, Eastwood R. A self-report scale predictive of drug compliance in schizophrenics: reliability and discriminative validity. Psychol Medicine 1983;13:177-83.

[5] Keith SJ, Kane JM. Partial compliance and patient consequences in schizophrenia: our patients can do better. J Clinical Psychiatry 2003;64:1308-15.

[6] Kikkert M, Schene A, Koeter M, Robson D, Gray R. Medication Adherence in Schizophrenia: Exploring Patients', Carers' and Professionals' Views. Schizophren Bull 2006;32:786-94.

[7] Lançon C, Auquier P, Llorca PM, Martinez JL, Bougerol T, Scotto JC. Psychometric properties of PANSS (Positive and Negative Syndrome Scale) in the French version in a sample of schizophrenic patients. Encéphale 1997;23:1-9.

[8] Marková IS, Jaafari N. L’insight en psychiatrie. Paris: Doin; 2008.

[9] Marcus SC, Olfson M. Outpatient antipsychotic treatment and Inpatient costs in Schizophrenia. Schizophren Bull 2008;34:173-80.

[10] Masand M, Roca M, Turner M, Kane J. Partial Adherence to Antipsychotic Medication Impacts the Course of Illness in Patients With Schizophrenia: A Review. Primary Care Companion. J Clinical Psychiatry 2009;11:147-54.

[11] Mohamed S, Rosenheck R, McEvoy J, Swartz M, Stroup S, Lieberman JA. Crosssectional and longitudinal relationships between insight and attitudes toward medication and clinical outcomes in chronic schizophrenia. Schizophr Bull 2009;35:336-46.

[12] Paillot C, Ingrand P, Millet B, Amador XF, Senon JL, Olié JP, Jaafari N. Insight Study Group. French translation and validation of the Scale to assess Unawareness of Mental Disorder (SUMD) in patients with schizophrenics. Encéphale 2010;36:472-7.

[13] Rocca P, Crivelli B, Marino F, Mongini T, Portaleone F, Bogetto F. Correlations of attitudes toward antipsychotic drugs with insight and objective psychopathology in schizophrenia. Compr Psychiatry 2008;49:170-6. 
[14] Rummel-Kluge C, Schuster T, Peters S, Kissling W. Partial compliance with antipsychotic medication is common in patients with schizophrenia. Aust N Z J Psychiatry 2008; 42:382-8.

[15] Velligan DI, Weiden PJ, Sajatovic M, Scott J, Carpenter D, Ross R, Docherty JP. Strategies for addressing problems with serious and persistent mental illness: recommendations from the expert consensus guidelines. Journal of Psychiatry Practice 2010;16:306-24. 
Tableau 1 : Caractéristiques de la population $(\mathrm{n}=98)$

\begin{tabular}{lc}
\hline Femmes & 50 \\
Age & $38,6 \pm 21,1$ \\
Nombre d'hospitalisations & $3,9 \pm 2,2$ \\
PANSS & $89,2 \pm 10,9$ \\
Symptômes positifs & $30,4 \pm 9.0$ \\
Symptômes négatifs & $28,2 \pm 6,9$ \\
Symptômes généraux & $30,4 \pm 6,6$ \\
Item G12 & $4,8 \pm 1,6$ \\
SUMD & $3,3 \pm 1,0$ \\
G1 & $3,2 \pm 1,1$ \\
G2 & $3,3 \pm 1,3$ \\
G3 & $6,3 \pm 3,3$ \\
IS score total & $4,1 \pm 2,4$ \\
IS2 & $4,3 \pm 2,9$ \\
IS3 & $-0,04 \pm 5,02$ \\
DAI score total & $1,35 \pm 3,26$ \\
Facteur 1 & Facteur 2
\end{tabular}


Tableau 2 : Sévérité de la maladie, Insight et Croyances relatives aux médicaments $(\mathrm{n}=98)$

\begin{tabular}{|c|c|c|c|}
\hline & Observant & Non observant & $\mathrm{p}$ \\
\hline Nombre de patients & 50 & 48 & \\
\hline PANSS & $86,2 \pm 11,87$ & $91,96 \pm 8,96$ & 0,005 \\
\hline Symptômes positifs & $27,94 \pm 9,49$ & $32,9 \pm 7,74$ & 0,01 \\
\hline Symptômes négatifs & $29,36 \pm 7,55$ & $27,04 \pm 5,97$ & 0,15 \\
\hline Symptômes généraux & $28,9 \pm 7,34$ & $32 \pm 5,28$ & 0,02 \\
\hline Item G12 & $4,26 \pm 1,63$ & $5,31 \pm 1,4$ & 0,001 \\
\hline \multicolumn{4}{|l|}{ SUMD } \\
\hline G1 & $2,96 \pm 1,05$ & $3,65 \pm 0,81$ & $<0,0001$ \\
\hline G2 & $2,84 \pm 1,15$ & $3,58 \pm 0,96$ & $<0,0001$ \\
\hline G3 & $2,96 \pm 1,32$ & $3,73 \pm 1,09$ & 0,003 \\
\hline IS & $7,22 \pm 3,44$ & $5,29 \pm 2,95$ & 0,001 \\
\hline IS2 & $4,64 \pm 2,32$ & $3,52 \pm 2,42$ & 0,017 \\
\hline IS3 & $5,08 \pm 2,97$ & $3,5 \pm 2,65$ & 0,006 \\
\hline DAI & $1,4 \pm 4,77$ & $-1,5 \pm 4,89$ & 0,005 \\
\hline Facteur 1 & $1,6 \pm 2,66$ & $1 \pm 3,05$ & 0,4 \\
\hline Facteur 2 & $-0,16 \pm 3,43$ & $-2,58 \pm 2,58$ & $<0,0001$ \\
\hline
\end{tabular}

